\title{
PREDICTION OF ELECTRIC PERMITTIVITY OF THREADS IN WOVEN FABRIC
}

\author{
Jacek Leśnikowski
}

Institute of Architecture of Textiles, Lodz University of Technology

Zeromskiego 116, 90-924 Lodz, Poland; jacek.lesnikowski@p.lodq1z.pl

\begin{abstract}
:
In the article, a new method for the estimation of electric permittivity of threads (filaments) was presented. The proposed recursive method is based on the results of computer simulation of $3 D$ model of transmission stripline. This model contains a model of flat fabric having threads, with which electric permittivity should be determined. The described procedure uses the method proposed by Barry to obtain permittivity of flat fabric from the so-called $s$ parameters of the simulated stripline. In the proposed method, the permittivity of the flat fabric obtained from simulation is compared with the measured value of permittivity of real flat fabric in order to estimate the threads' permittivity. This comparison is needed to obtain the electric permittivity of threads forming this fabric. The article also presents examples of the obtained values of threads' permittivity and discussion about the accuracy of the method. The presented method will be useful in situations where the knowledge of permittivity of threads is necessary in the conducted research.
\end{abstract}

\section{Keywords:}

threads electric permittivity, woven properties, dielectric properties of textiles, filaments permittivity

\section{Introduction}

In the conducted research, the knowledge of electric permittivity of threads forming a flat textile product is often needed, particularly in computer simulations of highfrequency electrical properties of textiles. The results of such simulations can be useful when designing textile antennas,[1] textile signal lines, $[2,3]$ textile humidity sensors, $[4,5]$ variable thermoinsulation garments,[6] and so on. The dielectric constant of threads creating a flat textile product (e.g., fabric) must be known, especially in situations when computer modeling is needed. The user of programs intended for high frequency simulation of electrical properties of flat textile products, often has a problem with the selection of the electric permittivity of threads forming fabric, in order for the permittivity of the simulated fabric to be the same as the actual permittivity of fabric for which the studies were performed. Measurement methods of material permittivity are widely described in the literature. $[7,8]$ A summary of these methods together with an assessment of their usefulness for the measurement of textile materials was presented, among others, in [9]. The presented methods most often allow to determine the permittivity of the whole fibrous structure (e.g., fabric) which may differ significantly from the permittivity of threads due to the presence of air spaces in the measured structure. Bal and Kothari[10] found that the developed methods for the measurement of permittivity of threads have a number of drawbacks. Resonance (cavitation) methods[11] allow, for example, the measurement of permittivity only for a well-defined frequency. Thus, in case of necessity of carrying out measurements in a wide range of frequencies, this method provides insufficient information.

Bal and Kothari[10] reviewed the measurement methods of the threads' permittivity proposed in the literature. They found that despite years of research conducted by researchers in the world, the development of measurement method characterized by a high degree of accuracy is still a problem. This has led to what Bal and Kothari[12] attempted to find, that based on theoretical considerations and accepted geometric structures of fabrics, functional relationship linking the permittivity of threads forming the fabric and permittivity of fabrics is determined by the capacitive method (described in ASTM D150 standard[13]). The method described in ASTM D150 standard[13] is based on the capacitance measurement of the flat capacitor with the tested fabric disposed between the plates and the capacity of the same capacitor with air between the plates. Despite ASTM D150 standard[10] being applicable for low frequency measurements (the frequency range addressed extends from less than $1 \mathrm{~Hz}$ to several hundred megahertz), many of the permittivity instruments available on the market using flat capacitor method measure this parameter up to a few $\mathrm{GHz}$. Dielectric constant of the sample can then be determined from:

$$
\varepsilon_{r m}=\frac{C_{t}}{C_{0}},
$$

where Co is the capacitance of the capacitor with air between the plates, Ct is the capacitance of the capacitor with the tested fabric disposed between the plates. For geometric considerations, Bal and Kothari[12] assumed three models imitating the structure of the fabric: equivalent solid film, model of the fabric made from two transversely arranged set of yarns, which are laid in two layers without any interlacement and cylindrical model with threads intertwined with each other. For the assumed models, they obtained the functional dependencies that enable the determination of threads permittivity, based on the knowledge 
of fabric permittivity determined by the capacitive method $\left(\varepsilon_{\mathrm{rm}}\right)$ and the selected geometrical parameters of the structure. Obtained dependencies were verified experimentally through the measurement of dielectric constants of physical models of fabrics and comparing them with values obtained from the functional dependencies. Physical models were weaved from the woven fabrics, the monofilaments of which were made from high-density polyethylene so as to ensure the possibility of precise control of the geometrical parameters of the structure. As a result of the verification, Ball and Kothari[12] found that only the model of cylindrical threads interwoven with each other and corresponding functional dependence may find practical application. However, also in this model, the differences between the values obtained from the functional dependencies and the values from the measurement of physical models reach $25 \%$ of the measured value. It can therefore be assumed that the existing determination methods of threads permittivity, based on functional dependency found by theoretical considerations, have low accuracy. The above considerations lead to the need to develop a new method for permittivity estimation of threads. Accordingly, a new method (procedure) for the determination of electric permittivity of threads was designed. This method is presented in the article below. The main novelty of the proposed method is to use the computer simulation to obtain complex permittivity of threads. The presented method will be useful in situations where the knowledge of permittivity of threads is necessary in the conducted research. For example, when designing different kinds of textile sensors, textile antennas or textile signal lines. The proposed method allows to answer the question: what should be the permittivity of the threads so that the fabric obtained from them has an assumed permittivity?

\section{Description of the method for estimation of threads permittivity in fabric}

The developed method for determination of threads permittivity in fabric is based on values of dielectric constant $\varepsilon_{\mathrm{rm}}$ and loss tangent tgd of this fabric. These parameters are determined from the measured capacitance of fabric.

The proposed method uses CST MICROWAVE STUDIO $尺$ simulation software to determine the so-called $s$ parameters of the transmission line with the inserted, tested fabric. More information on these parameters can be found in $[3,14-16]$.
CST MICROWAVE STUDIO ${ }^{\circledR}$ based on the finite element method and Maxwell's equations enables the fast and accurate analysis of the high frequency (HF) 3-D devices. To obtain all the presented simulations, the time domain solver was used with a hexahedral mesh.

On the basis of $s$ parameters, based on the method proposed by Barry[17] and using the method proposed in the article, the complex permittivity of fabric can be determined and from there, the permittivity of threads that create this fabric.

In the method proposed by Barry,[17] complex permittivity of the tested material can be determined by the measurement of $s$ parameters of the signal line shown in Figure 1. The tested material is placed in the middle of the line, above and below the inner signal path.

Line shown in Figure 1 is a typical stripline with inserted two pieces of material with which permittivity should be determined. The line can be thought as a serial connection of two air striplines with a length $I p$ and a stripline with a length $d p$ between them filled with a dielectric material of unknown permittivity. If we denote the characteristic impedance of air stripline by $Z 0$, the impedance of stripline filled by the tested material is:

$$
Z=Z_{0} \sqrt{\frac{\mu_{r}}{\varepsilon_{r}}} .
$$

In formula (2), $\mu r$ is a complex magnetic permeability and $\varepsilon r$ is a complex permittivity of the material under test.

For line segments that are not filled by the tested material, the propagation constant can be expressed as:

$$
\gamma_{0}=2 \pi f \sqrt{\mu_{0}},
$$

where $f$ is frequency of electromagnetic wave, $\mu_{0}, \varepsilon_{0}$ are magnetic and electric permittivity of vacuum respectively.

Filling the middle part of the stripline with material for which permittivity

$$
\operatorname{Re}\left(\varepsilon_{r}\right) \neq 1
$$

causes that characteristic impedance of the line is different

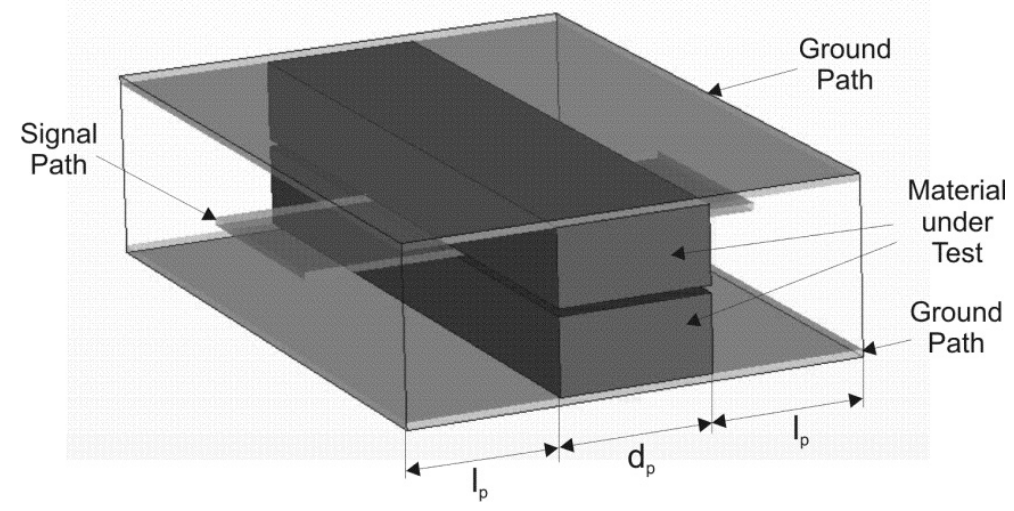

Figure 1. The signal line proposed by Barry[14] to measure the electric permittivity 
from the impedance of the line segments filled with air located at the beginning and end of the line. This leads to reflections of the wave propagating through the line, on the boundary between the line segment filled with air and a line segment filled with tested material. The reflection coefficient can be expressed by:

$$
R=\frac{Z-Z_{0}}{Z+Z_{0}}
$$

From equations (2), (3) and (5), we can obtain:

$$
\varepsilon_{\mathrm{r}}=\frac{\gamma(1-\mathrm{R})}{\gamma_{0}(1+\mathrm{R})} \text {. }
$$

Knowledge of propagation constant $\gamma, \gamma_{0}$ and reflection coefficient $R$, enables the determination of complex permittivity of the tested material.

Barry,[17] in his measurement method, uses the well-known Collin's theory,[18] which allows one to find the relationship between the coefficients $\gamma, \gamma_{0}, R$ and the values of the $s$ parameters that are measured for the structure shown in Figure 1.

To find such a relationship, the two-port model of the line from Figure 1 should be adopted and relationships occurring between incident and reflected waves for both sides of this twoport model should be considered. These relationships can be written as [17]:

$$
\gamma=\frac{\arccos \left(\frac{e^{-j 4 \gamma_{0} l} p_{+S_{12}}^{2}-S_{11}^{2}}{2 e^{-j 2 \gamma_{0} l_{p}}}\right)}{d_{p}}
$$

and

$$
R=\frac{S_{11}}{w^{-j 2 \gamma_{0} l_{p}}-S_{12} e^{-j \gamma d_{p}}}
$$

In summary, on the basis of the measured $s$ parameters, from equations (3), (6), (7) and (8), the value of the complex permittivity of the material placed in the middle of the stripline (Figure 1) can be obtained. The main advantage of this method is that the obtained complex permittivity allows the evaluation of the loss in the tested material (loss tangent coefficient tgd). The method of calculation described above was used for the determination of permittivity of threads in the following test procedure. Due to the presence of air pores in flat textiles (using the method proposed in [17]), we obtain the value of the effective permittivity. The knowledge of this permittivity is necessary to determine the permittivity of threads forming the flat textile according to the proposed procedure. Textiles exhibit an anisotropy of their properties, for example, the anisotropy of resistance.[19] Despite the fabric anisotropy, the method can be used to determine the electric permittivity for the different (assumed) direction of the fabric to be tested.

The developed procedure can be used to determine the permittivity of both yarns and threads forming the flat textile material, depending on the adopted geometric 3D structure of that material. This procedure uses the value of the relative permittivity of the fabric and consists of several steps, as shown in a diagram in Figure 2.
As can be seen from Figure 2, the proposed procedure involves creating a computer 3D structure of the fabric with preassumed value of permittivity of threads that create it. Then, we repeatedly simulate the transmission properties of the line (Figure 1) with a placed piece of fabric created according to the adopted structure. The goal of each simulation is to obtain $s$ parameters of the simulated line. In the next step, permittivity of fabric should be computed from the obtained $s$ parameters. If the computed value of permittivity does not differ from the value obtained from measurement more than a predetermined maximum difference, the procedure ends and should be assumed that pre-assumed permittivity of threads corresponds to the real values. Otherwise, other permittivity values of threads should be assumed, and the procedure should be repeated.

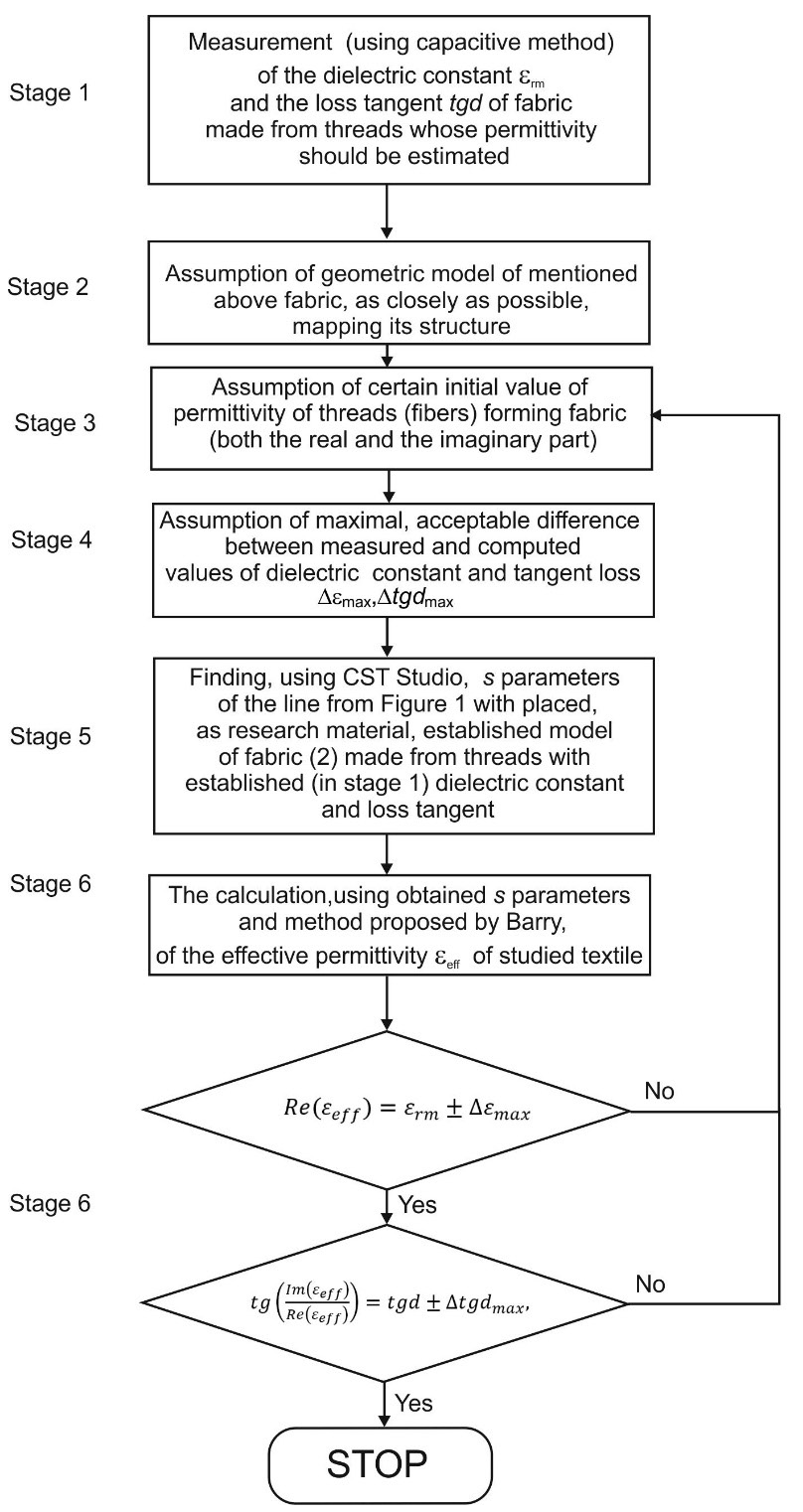

Figure 2. Procedure flowchart for the estimation of threads' permittivity

\section{Accuracy of the proposed method}

The accuracy of calculated permittivity of threads, according to the proposed procedure, depends on: the accuracy of measurements performed in step 1 (Fig.2), assumed (in step 2) model mapping the structure of the flat textile, the accuracy 
of the simulation performed in step 4 and the accuracy of the method proposed by Barry.[17] Verification of the proposed method by comparison of threads permittivity obtained from the proposed method with values obtained from the measurement is very difficult. Using the flat capacitor method, the electric permittivity could be measured for up to $1 \mathrm{GHz}$. Because the value of the electric permittivity is frequency dependent, that is, all measurements and simulations should be done at the same frequency. The measurement of permittivity of threads using the cavity method[19] with metal, rectangular, microwave cavity working at $\mathrm{TE}_{10 \mathrm{~N}}$ odd modes at $1 \mathrm{GHz}$ requires $12,800,000 \mathrm{~mm}^{3}$ of cavity. The dielectric constant determined by a the rectangular cavity is expressed by the given equation[20]:

$$
\varepsilon_{\mathrm{r}}=1+0,5 \cdot \frac{\mathrm{f}_{\mathrm{c}}-\mathrm{f}_{\mathrm{s}}}{\mathrm{f}_{\mathrm{s}}} \cdot \frac{\mathrm{v}_{\mathrm{c}}}{\mathrm{v}_{\mathrm{s}}},
$$

where $c$ is the resonant frequency of empty cavity, $f_{s}$ is resonance frequency of the cavity with disposed sample, $V_{c}$ is volume of the cavity and $V s$ is the volume of the sample. From equation (9), we can obtain that:

$$
\mathrm{f}_{\mathrm{s}}=\mathrm{f}_{\mathrm{c}} \cdot\left(1+\frac{2 \cdot\left(\varepsilon_{\mathrm{r}}-1\right) \cdot \mathrm{v}_{\mathrm{s}}}{\mathrm{v}_{\mathrm{c}}}\right)
$$

Assuming $f_{c}=1 \mathrm{GHz}$ (maximum usable frequency for permittivity measurement using flat capacitor method), $V_{c}=12,800,000 \mathrm{~mm}^{3}$ and assuming that the diameter of the measured yarn is $1 \mathrm{~mm}$ and its length is equal to the height of the resonant cavity, placing it to the resonant cavity will cause a relative change in the resonance frequency only about $0.005 \%$. Measurement of thinner yarns or individual fibers causes an even smaller change in frequency. The measurement of such small frequency change is subject to considerable uncertainty. This causes that use cavity method for measuring the permittivity of yarns (filaments) at frequencies lower than several $\mathrm{GHz}$ is practically impossible. On the other hand, as noted above, the maximum usable frequency for permittivity measurement using the flat capacitor method is about $1 \mathrm{GHz}$. This makes it impossible to verify the presented method by measuring the permittivity of the thread by the resonant cavity method. Due to the problems described above with the measurement of threads permittivity, verification of the accuracy of the proposed method was limited to checking the accuracy of Barry's method[17] and the accuracy of the simulation. It has been verified by simulation of operation of the line shown in Figure 1, with the tested material placed in the form of a cuboid with assumed values of dielectric permittivity and loss tangent. After determining, using CST MICROWAVE STUDIO ${ }^{\circ}$, the values of $s$ parameter of the line, using method proposed by Barry,[17] the value of electric permittivity and loss angle test material were calculated. The results in the form of relative deviations between the calculated and assumed values are shown in Figures 3 and 4.

Figure 3 shows the relative deviations between the determined and assumed values of permittivity $\varepsilon r$ for various values of loss tangent of the tested material. In turn, Figure 4 shows the relative deviation between the obtained and assumed loss tangent values for assumed different values of dielectric constant.

\section{Results and discussion}

In order to further verify the method, a few real fabrics were selected. Next, the computer models, imitating their thickness, type of weave, diameter of the weft and the warp, weft and warp density, were made. Threads in the constructed models had the form of monofilaments with ellipsoidal cross-sectional area. Each model was combined with stripline form, creating the structure shown in Figure 5.

Then, using the method described in the article, the electric permittivity of threads forming the fabrics were determined. The values of permittivity of fabrics obtained by measurement using a flat capacitor method and the obtained permittivity of threads forming this fabric are shown in column 6 of Table 1. The values of loss tangent of the fabrics measured by the flat capacitor method and the values of loss tangent of the yarns forming these fabrics are shown, in Table 1 (column 7).

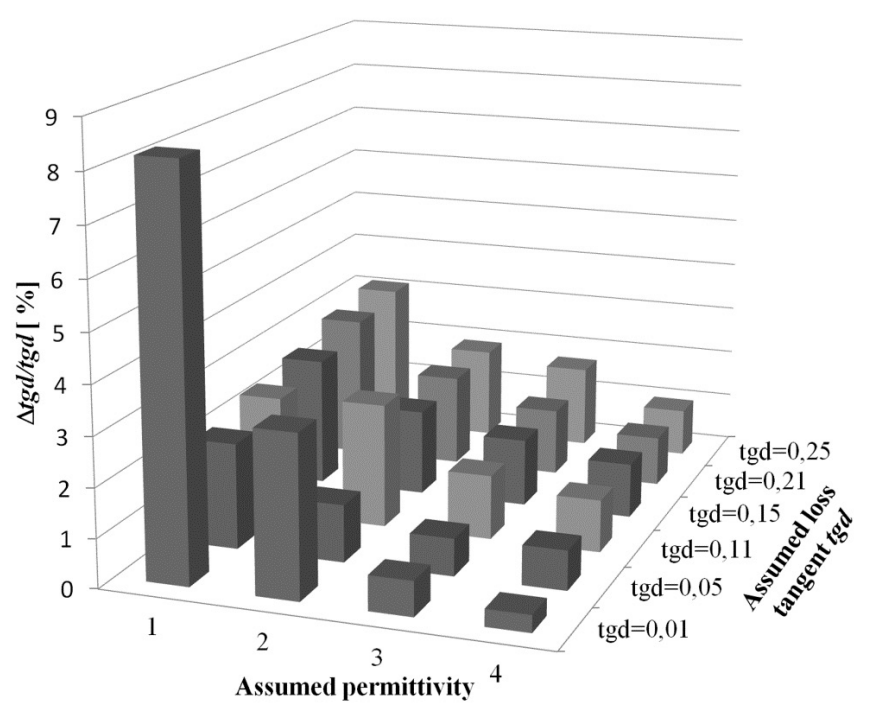

Figure 3. The relative deviation between the dielectric constant determined using Barry's method ${ }^{[17]}$ and the assumed values of dielectric constant and loss tangent tgd

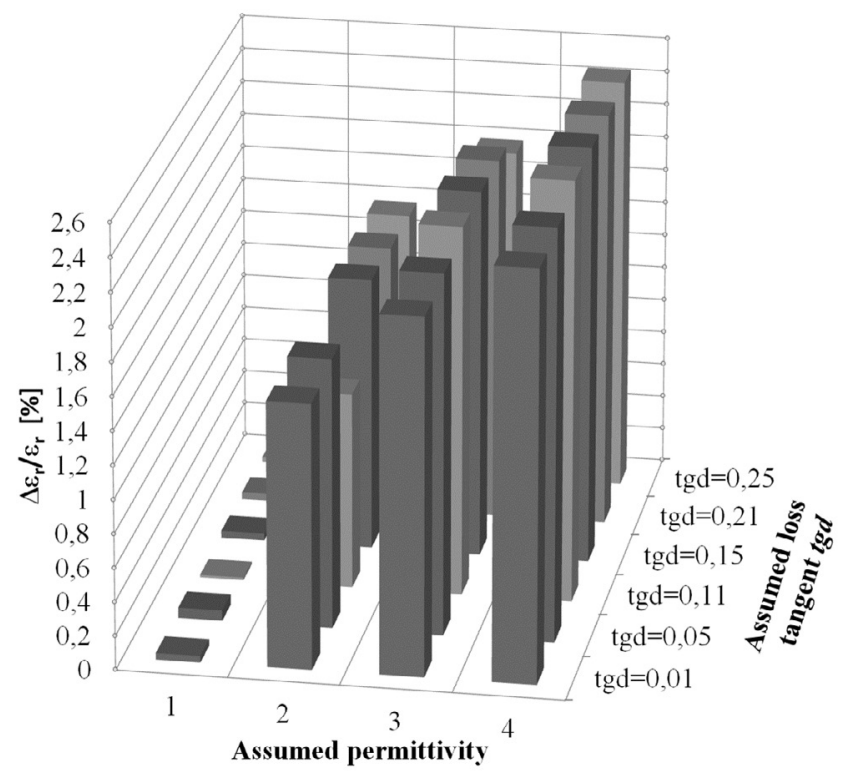

Figure 4. The relative deviation between the loss tangent determined using Barry's method and the assumed values of constant loss tangent tgd and dielectric constant 


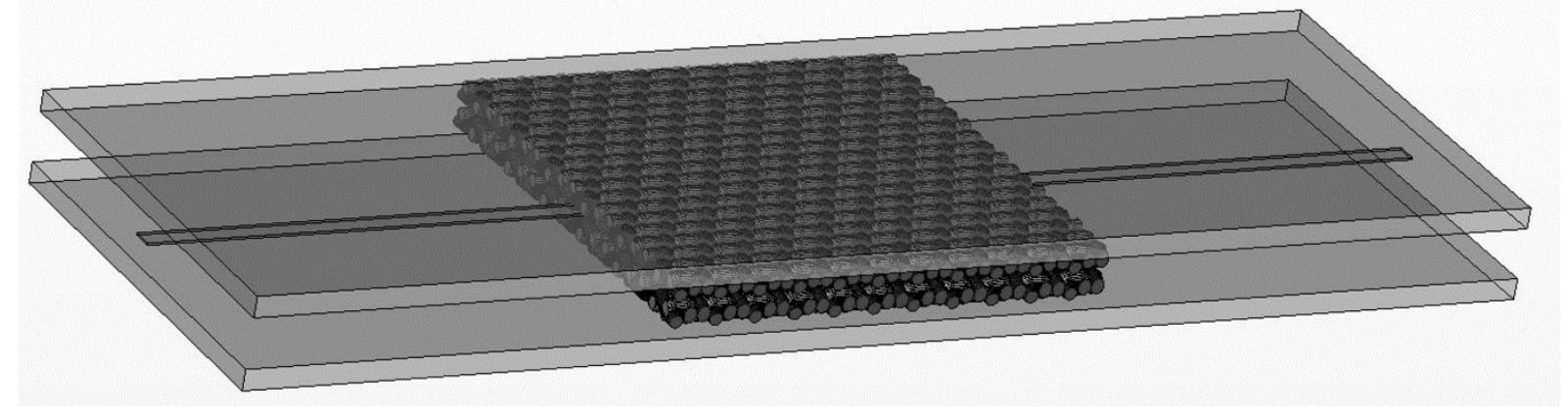

Figure 5. Model of the transmission line with inserted, tested material in the form of fabric

Table 1. Permittivity of fabrics and permittivity of threads forming these fabrics obtained from the proposed method

\begin{tabular}{|c|c|c|c|c|c|c|}
\hline Material & Weave & Thickness & $\begin{array}{c}\text { Density of } \\
\text { warp }\end{array}$ & $\begin{array}{c}\text { Density of } \\
\text { weft }\end{array}$ & $\begin{array}{c}\text { Electric } \\
\text { permittivity of } \\
\text { fabric/thread } \\
(\mathrm{f}=1 \mathrm{GHz})\end{array}$ & $\begin{array}{c}\text { Loss tangent of } \\
\text { fabric/thread }(\mathrm{f}=1 \\
\text { GHz) }\end{array}$ \\
\hline- & - & $\mathrm{mm}$ & threads/cm & threads/cm & - & - \\
\hline $100 \%$ polyester & Satin & 0,48 & 82 & 30 & $1,326 / 1,886$ & $0,003 / 0,003$ \\
\hline $\begin{array}{c}75 \% \text { cotton } \\
24 \% \text { polyester } \\
1 \% \text { antistatic fibers }\end{array}$ & Twill & 0,55 & 32 & 20 & $2,471 / 3,705$ & $0,03 / 0,05$ \\
\hline $100 \%$ linen & Plain & 0,42 & 24 & 24 & $1,730 / 2,264$ & $0,070 / 0,073$ \\
\hline $\begin{array}{c}93 \% \text { polyester } \\
7 \% \\
\text { elastomer }\end{array}$ & Satin & 0,22 & 55 & 141 & $1,252 / 1,446$ & $0,007 / 0,009$ \\
\hline
\end{tabular}

\section{Conclusions}

The method presented in the article allows the determination of the electric permittivity of threads forming fabric based on the permittivity of the fabric determined by capacitive method (IEC 250:1969 [21]). Figures 3 and 4 show that Barry's method,[17] in conjunction with the simulation conducted in CST MICROWAVE STUDIO $®$ program, aiming to achieve the values of $s$ parameters of the measuring line does not significantly affect the accuracy of the designation of permittivity of the yarns. The accuracy of the presented method depends mainly on the quality of the adopted geometric structure of the flat textile product. The exact determination of this relationship is difficult and requires further study.

\section{References}

[1] Roshni S. B. et al. (2017). Design and fabrication of an E-shaped wearable textile antenna on PVB-coated hydrophobic polyester fabric, Smart Mater. Struct., 26, 1 - 8.

[2] Leśnikowski J. (2015). New Kind of Textile Transmission Line with an Impedance of 50 Ohms. Fibres \& Textiles in Eastern Europe, 23(2), 51-54.

[3] Leśnikowski J. (2011). Textile Transmission Lines in the Modern Textronic Clothes, FIBRES \& TEXTILES in Eastern Europe, 19(6), 89-93.

[4] Zhou G. et al. (2017). Highly Sensitive Wearable TextileBased Humidity Sensor Made of High-Strength, SingleWalled Carbon Nanotube/Poly(vinyl alcohol) Filaments, ACS Appl. Mater. Interfaces, 9(5), 4788-4797.
[5] Kubiak P., Leśnikowski J. Gniotek K. (2016). Textile Sweat Sensor for Underwear Convenience Measurement. Fibres \& Textiles in Eastern Europe, 24(6),151-155.

[6] Kurczewska A., Leśnikowski J. (2008). Variablethermoinsulation garments with a microprocessor temperature controller, International Journal of Occupational Safety and Ergonomics, 14 (1), 77-87.

[7] Zhang H., Zeng B.Q., Ao L., \& Zhang Z. (2012). A novel dual-loop coupler for one-port Cylindrical cavity permittivity measurement, Progress in Electromagnetics Research, 127, 537-552.

[8] Bappadittya R., Bhatterchya A.K., \& Choudhury S. K. (2013, December). Characterization of Textile Substrate to Design a Textile Antenna, Paper presented at the International Conference on Microwave and Photonics (ICMAP), Dhanbad, India.

[9] Leśnikowski J. (2012). Dielectric permittivity measurement methods of textile substrate of textile transmission lines, Electrical Review, 3a, 148-151.

[10] Bal K., \& Kothari V.K. (2009). Measurement of dielectric properties of textile materials and their applications, Indian Journal of Fibre \& Textile Research, 34, 191-199.

[11] Kumar A., \& Sharma S. (2007). Measurement of dielectric constant and measurement of dielectric constant and loss factor of the dielectric material at microwave frequencies, Progress In Electromagnetics Research, PIER 69, 47-54.

[12] Bal K., \& Kothari V.K. (2009). Study of dielectric behavior of woven fabric based on two phase models, Journal of Electrostatics, 67, 751-758. 
[13] ASTM D 150-11. (2011). Standard test methods for a-c loss characteristics and permittivity (dielectric constant) of solid electrical insulation. Retrieved February 02, 2016, from http://www.astm.org/Standards/D150.htm

[14] Radmanesh M. (2007). RF \& Microwave Design Essentials: Engineering Design and Analysis from DC to Microwaves. Bloomington: AuthorHouse.

[15] Hiebel M. (2007). Fundamentals of Vector Network Analysis. Munich: Rohde-Schwarz.

[16] Ellinger F. (2007). Radio Frequency Integrated Circuits and Technologies. Berlin Heidelberg: Springer.

[17] Barry W. (1986). A Broad-Band, Automated, Strip line Technique for the Simultaneous Measurement of Complex Permittivity and Permeability, IEEE Transactions on Microwave Theory and Techniques, MTT34, 1, 80-84.
[18] Collin R. E. (1960). Field Theory of Guided Waves. New York: McGraw-Hill, 3, 79-83.

[19] Tokarska M., Gniotek K. 2015. Anisotropy of the electrical properties of flat textiles, The Journal of The Textile Institute, 106(1), 9-18.

[20] Sheen J. (2007). Amendment of cavity perturbation technique for loss tangent measurement at microwave frequencies, Journal of Applied Physics, 102, 014102-1 014102-6.

[21] IEC 250:1969, Recommended methods for the determination of the permittivity and dielectric dissipation factor of electrical insulating materials at power, audio and radio frequencies including meter wavelengths. Retrieved February 02, 2016, from https://webstore.iec.ch/ publication $/ 1151 \&$ preview $=1$ 